\title{
Target-tilt and vertical-hemifield asymmetries in free-scan search for 3-D targets
}

\author{
FRED H. PREVIC \\ Air Force Research Laboratory, Brooks Air Force Base, Texas \\ and \\ PETER D. NAEGELE \\ Research and Development Laboratories, Culver City, California
}

\begin{abstract}
In this study, asymmetries in finding pictorial 3-D targets defined by their tilt and rotation in space were investigated by means of a free-scan search task. In Experiment 1, feature search for cube tilt and rotation, as assessed by a spatial forced-choice task, was slow but still exhibited a characteristic "flat" slope; it was also much faster to upward-tilted cubes and to targets located in the upper half of the search field. Faster search times for cubes and rectangular solids in the upper field, an advantage for upward-tilted cubes, and a strong interaction between target tilt and direction of lighting (upward or downward) for the rectangular solids were all demonstrated in Experiment 2. Finally, an advantage in searching for tilted cubes located in the upper half of the display was shown in Experiment 3, which used a present-absent search task. The results of this study confirm that the upper-field bias in visual search is due mainly to a biased search mechanism and not to the features of the target stimulus or to specific ecological factors.
\end{abstract}

Most previous research concerning visual search in humans has used targets defined by two-dimensional (2-D) features, such as size, shape, and color, which are generally most useful in object search and recognition. Most studies using a free-scan procedure have shown that such targets are found more quickly and accurately in the upper and right hemifields (see the review by Previc, 1998). One explanation for this upper-right advantage is that it reflects a fundamental linkage of object search and scanning to focal extrapersonal attentional mechanisms, which are hypothesized to be biased in these same directions (see Previc, 1998).

Several studies have also examined the nature of visual search to pictorial three-dimensional (3-D) targets defined by their orientation in space (Enns \& Rensink, 1990, 1991; Humphreys, Keulers, \& Donnelly, 1994; Sun \& Perona, 1996; von Grünau \& Dubé, 1994). Although some researchers believe that a robust 3-D appearance leads to faster and more efficient search for solid targets than for 2-D figures, evidence of a 3-D advantage remains somewhat equivocal. On the one hand, Sun and Perona

This study was supported in part by a grant from the Air Force Office of Scientific Research (AFOSR) to the first author and an AFOSRfunded summer fellowship to the second author. The views expressed in this paper do not necessarily reflect the views of the United States Air Force or the Department of Defense. We thank Joe Campbell, Bob Gallaway, and Kevin Nuse for their computer support, Jeremy Beer and Marc Green for their comments on the manuscript, and Brenda Cobb, Joe Fischer, and Carolyn Oakley for their statistical support. Correspondence concerning this article should be addressed to F. H. Previc, TASC, Inc., 4241 Woodcock Dr., Ste. B100, San Antonio, TX 78228 (email: fred.previc@brooks.af.mil). showed that the stimulus duration required for finding 3-D shapes oriented in a particular direction is independent of the number of distractors, unlike the case for arbitrary 2-D shapes composed of the same elements. However, the target shape yielding the fastest and most efficient search times in Sun and Perona's study was much less 3-D in appearance than others whose detectability was greatly slowed by increasing the number of distractors. ${ }^{1}$ Moreover, the results of at least one study (Brown, Weisstein, \& May, 1992) indicate that searching for 3-D shapes may even be slower and more dependent on distractor set size than searching for distinctive 2-D cues.

Visual field asymmetries in search for 3-D shapes have also proven to be more complex than in the case of 2-D shapes. For example, von Grünau and Dubé (1994) showed that upward-tilted targets embedded among downwardtilted distractors are found more quickly in the lower visual field than in the upper visual field, whereas no significant vertical hemifield differences were reported in searching for downward-tilted targets. Von Grünau and Dubé also reported overall faster reaction times (RTs) in the lower visual field, although this trend actually proved significant only in Experiment 4.2 of their study. These authors interpreted the lower-visual-field advantageand particularly, the specific advantage of upward-tilted cubes in the lower visual field -in terms of the ecological prevalence of seeing 3-D cubes from above. According to this hypothesis, a downward-tilted distractor field in the lower visual field is more aligned with the ground plane as viewed at an elevated angle, so that the presence of an oppositely tilted (i.e., upward) target is much more salient than when the tilts of the targets and the distractors are 
reversed (von Grünau \& Dubé, 1994). However, search times for downward-tilted cubes among upward-tilted distractor cubes lit from below actually improve when the latter are placed along a simulated "floor" (Sun \& Perona, 1996), which suggests that the advantage of the upwardtilted cubes in the lower visual field is not due solely to the simulated downward view-angle of the subject.

The main purpose of the present study was to determine whether 3 -D targets defined by their spatial properties are more quickly found in the upper field (UF) —as in previous free-scan studies that varied the size and shape of 2-D search targets (e.g., Previc, 1996; Previc \& Blume, 1993) — or in the lower field (LF), as was found by von Grünau and Dubé (1994). In all of the experiments described in this paper, a free-scan procedure was used so that UF and LF will refer to the locus of the target relative to the center of the search display and relative to the fixation point when the search field first appeared. (After a saccadic scanning movement occurred, the target's vertical hemifield, relative to the new fixation point, varied according to the saccade's direction.) Two features were manipulated in Experiment 1: (1) 3-D tilt, which was used by Enns and Rensink (1990), Sun and Perona (1996), and von Grünau and Dubé, and (2) rotation in depth, which has an advantage over other motion cues in that the moving target retains its same overall position in the search display. In order to compare the vertical-hemifield asymmetries obtained with tilt and rotation with those obtained with such 2-D cues as size and color, the visual search conditions and tasks were, aside from the different target parameters and minor differences in overall display size and luminance, virtually identical to those of Previc (1996) and Previc and Blume. A major aim of Experiment 2 was to vary the direction of lighting for stationary cubes and rectangular solids, in order to further determine how much vertical-field and target asymmetries are influenced by ecological factors. Finally, a present-absent search task was compared with a spatial forced-choice search task in Experiment 3 to determine whether the opposite vertical asymmetries obtained in previous studies might have been due to the type of search task per se.

\section{EXPERIMENT 1 \\ Vertical-Hemifield and Target Asymmetries in Feature Search for Cube Tilt and Rotation}

The previous literature concerning search for the spatial properties of 3-D shapes has focused mainly on the orientation of the shape in 3-D space (usually tilted upward or downward). Whether or not efficient search for these features occurs has been shown to be dependent on the relative appearance of the target and the distractor fields; for example, searching for a particular 3-D tilt of a "solid" cube is only slightly influenced by the size of the distractor set (Enns \& Rensink, 1990; Sun \& Perona, 1996), whereas searching for the tilt of wire-frame cubes or other 3-D solid shapes is much more affected (Sun \& Perona, 1996; von Grünau \& Dubé, 1994).
In Experiment 1, subjects searched for cubes defined by their 3-D tilt or rotation. In the tilt-search condition, solid cubes were tilted either upward or downward against variously sized distractor fields of the opposite tilt, whereas target cubes moved in opposite horizontal rotation to the distractor field in the rotation-search condition. In addition to testing for possible vertical-field differences, a major objective of Experiment 1 was to determine whether feature search for tilt and rotation is performed independently of distractor set size, as is the case for salient 2-D features.

\section{Method}

Subjects. A total of 12 subjects, 9 of whom were male, participated in Experiment 1. The age of the subjects ranged from 24 to 51 years, with a mean age of 34.2 years. All the subjects were either full-time or visiting civilian and military personnel at Brooks Air Force Base. None had participated in a previous visual search experiment, and all were naive as to the hypotheses of the study. Each subject had 20/30 visual acuity or better, with or without correction, in each eye.

The voluntary, informed consent of the subjects was obtained as required by Air Force Instructions 40-402 and 40-403.

Stimuli and Apparatus. The stimuli were rotating cubes that were generated on a Silicon Graphics IRIS 3130 computer and presented on an Hitachi $60-\mathrm{Hz}$ color video monitor (Model CM2086A1SG). Each cube consisted of six 1.0-cm sides of varying luminances: two opposite dark gray sides, two opposite light gray sides, and light top and bottom faces (Figure 1). Hence, the average luminance of the cubes varied, between 2.33 and $3.79 \mathrm{~cd} / \mathrm{m}^{2}$, depending on which side faced the subject. The cubes also differed in their tilt in pitch, relative to the subject- $25^{\circ}$ downward with the top face visible, or $25^{\circ}$ upward with the bottom face shown. (The opposite vertical brightness gradients for the two cube types ensured that their overall luminances would be the same.) In all the conditions, the cubes started from the same forward position and rotated either leftward or rightward at $30 \% \mathrm{sec}$ through their center axes, with an average frame update rate of approximately $14 \mathrm{~Hz}$. Thus, the cube's maximum displacement was $90^{\circ}$, if the search field remained on for its full 3 -sec interval. The average luminance of the background was $0.07 \mathrm{~cd} / \mathrm{m}^{2}$.

The search field consisted of a single target and a total of 11,23, or 35 evenly dispersed distractors in the low-density, medium-density, and high-density distractor conditions, respectively. This ensured that 3,6 , or 9 distractors appeared per quadrant, with a single distractor removed from one of the four quadrants to allow for the presentation of the target cube. All the stimuli were located in the plane of fixation and in three different eccentricity rings: $2.1^{\circ}, 3.6^{\circ}$, and $5.6^{\circ}$. The overall diameter of the circular search field was $12.3^{\circ}$, which was comparable with that of Previc (1996, Experiment 2). The subjects viewed the stimuli at a distance of $81.3 \mathrm{~cm}$, identical to that of Previc (1996), while their forehead and chin rested in an ophthalmologic viewing brace. As they viewed the stimuli, the subjects sat in a darkened room in a comfortable chair that was adjustable in height.

As in Previc and Blume (1993) and Previc (1996), the subjects made an RT response when they found the target shape by depressing the right key on a Logitech Model C7 mouse, which was attached to a Zenith Z-248 personal computer (PC) whose clock had $\sim 1$-msec resolution. After making the RT response, the subjects registered the target's location by moving and setting a red cursor on the video monitor, using a second mouse (Mouse Systems Model 4Q) that was attached to the IRIS computer.

Task and Overall Procedure. The subjects performed a feature-search task in which the target differed by only a single fea- 


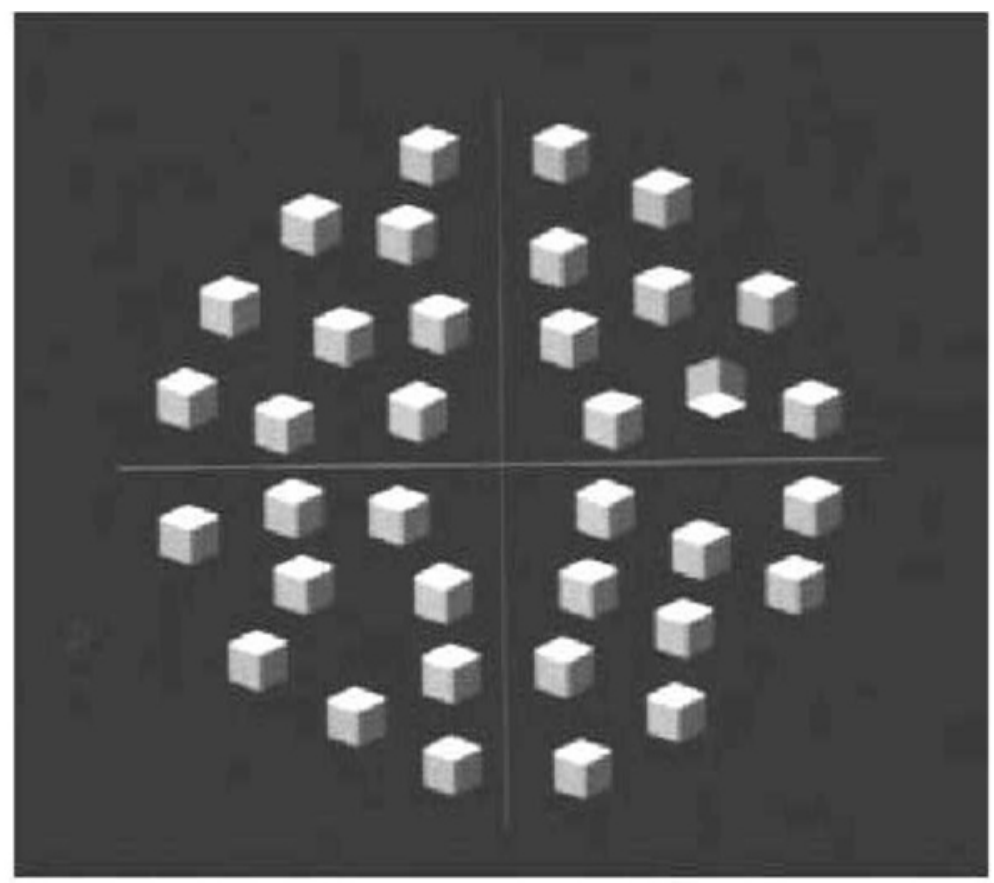

Figure 1. An illustration of the search field used in Experiment 1. The tilt condition with a full field ( 1 target and 35 distractors) is shown. The target cube (located in the upper-right quadrant) is tilted "upward," whereas all of the others are tilted "downward."

ture (tilt or rotation) from the background field of distractors. An individual trial began with a 1 -sec presentation on the video monitor of a centrally located circle with a diameter of $2.5^{\circ}$, which was then followed by the appearance of the circle and an enclosed small fixation cross for $500 \mathrm{msec}$. The fixation cross and circle were then replaced for $200 \mathrm{msec}$ by one of the four cube stimuli-upward- or downward-tilted cubes that rotated leftward or rightward-that served to cue the target for that trial. (The unpredictability of the cue and the explicit fixation instructions were designed to keep the subject's fixation in the center of the display until the search field appeared.) The search field was presented immediately after the disappearance of the cue and remained on for as long as it took the subject to find the target, using a free-scan strategy, up to a maximum of $3 \mathrm{sec}$. The subjects indicated whether they had located the target (which was present on all trials) by pressing the PC mouse key, using the index finger of the left hand. This RT response, if made within the allotted 3-sec interval, was followed by the presentation of a display that contained four empty quadrants. The subject's task was to move and set the cursor from its initial position in the center of the display to the quadrant in which the target appeared. This response was made by the index finger of the right hand, using the left key on the IRIS mouse. The subject had as long as $2 \mathrm{sec}$ to register what was essentially a four-alternative spatial forced-choice (4ASFC) RT response, performed in two stages.

A visual search trial block consisted of a total of 144 trials. Each of the four target cubes appeared three times in each eccentricity ring in each quadrant throughout a trial block, using a pseudorandom sampling-without-replacement sequence of targets and target locations. The subject could pause the trial sequence at any time by pressing the rightmost key on the IRIS mouse. A trial block typically required about $10 \mathrm{~min}$ to complete.
The entire experiment consisted of four 30-45 min sessions, each of which included three trial blocks/conditions. The first two sessions contained practice blocks, with all six conditions (three distractor density levels for each of the two features) being presented. The next two sessions contained three test blocks each, with the order of the six search conditions counterbalanced across the 12 subjects, using a Latin-square procedure.

\section{Results}

Repeated measures analyses of variance (ANOVAs) were used to analyze the results for each of two measures-RT latency on trials in which the target was correctly located (speed) and percentage of trials in which the target was correctly located (accuracy). In these ANOVAs, feature (tilt vs. rotation), vertical hemifield (upper vs. lower), and distractor density (low, medium, and high) were the within-subjects factors. An additional repeated measures ANOVA was performed to determine whether RTs in the UF and LF were different for upwardversus downward-tilted targets, regardless of whether tilt or rotation was the relevant feature for that trial.

The mean search times for tilt and rotation in the UF and LF as a function of distractor density level are shown in Figure 2. For the tilt feature (left panel), mean RTs across the three distractor densities ranged from 1,089 to $1,106 \mathrm{msec}$, with an average UF advantage of $129 \mathrm{msec}$. For the rotation feature (right panel), mean RTs ranged from 1,366 to $1,428 \mathrm{msec}$, with an identical mean UF ad- 
TILT

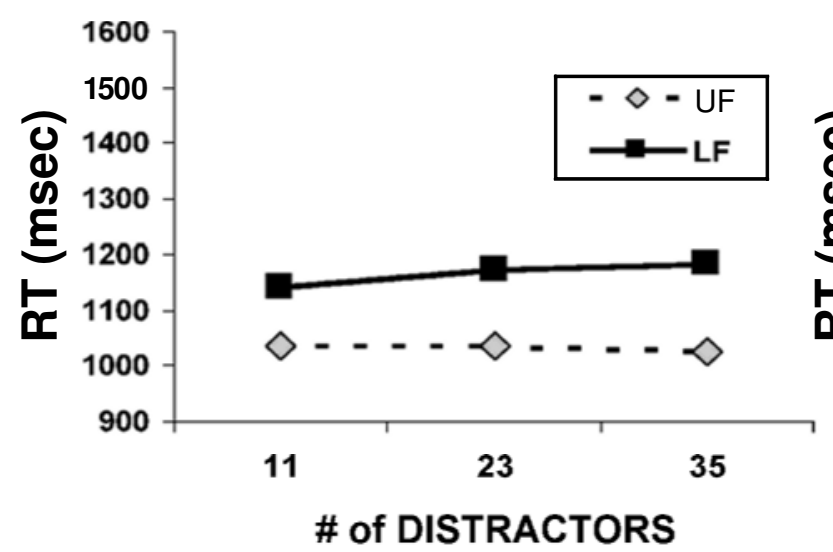

ROTATION

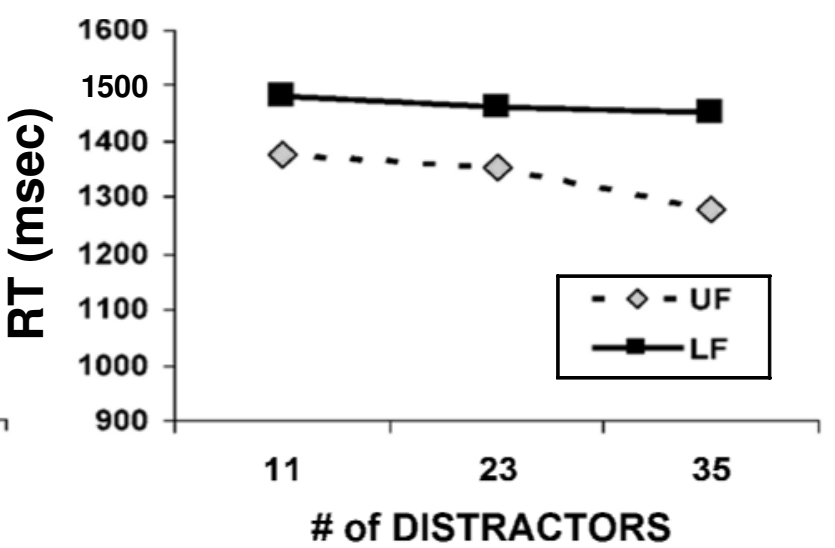

Figure 2. Mean reaction time (RT) latencies to upper field (UF, dashed lines) and lower field (LF, solid lines) targets in the tilt (left panel) and rotation (right panel) conditions of Experiment 1. The UF and LF RTs are shown for all distractor density levels (11, 23, and 35).

vantage of $129 \mathrm{msec}$. The RT ANOVA reflected the faster search times to both tilt-defined and UF targets, since significant main effects of feature $[F(1,11)=102.62, p<$ $.001]$ and hemifield $[F(1,11)=12.37, p<.01]$ were found. No other main effects or interactions were revealed by the speed ANOVA.

The UF and LF accuracy means for tilt and rotation as a function of distractor density level are shown in Figure 3. For the tilt feature (left panel), the means of the percentages of correct location responses ranged from $94.4 \%$ to $95.8 \%$, with an average UF advantage of $2.6 \%$. For the rotation feature (right panel), the means of the percentages of correct location responses ranged from $90.1 \%$ to
$91.3 \%$, with an average UF advantage of $3.7 \%$. The accuracy ANOVA reflected the greater percentage of correct location responses to both tilt-defined and UF targets, as significant main effects of feature $[F(1,11)=14.3, p<$ $.01]$ and hemifield $[F(1,11)=9.75, p<.01]$ were found. No other main effects or interactions were revealed by the accuracy ANOVA.

A final ANOVA sought to determine whether any differences between RTs to upward- and downward-tilted targets were present either overall or in the UF and LF individually. This ANOVA revealed significant main effects of direction of tilt $[F(1,11)=27.98, p<.001]$ and hemifield $[F(1,11)=12.37, p<.01]$, as well as a signif-

\section{TILT}

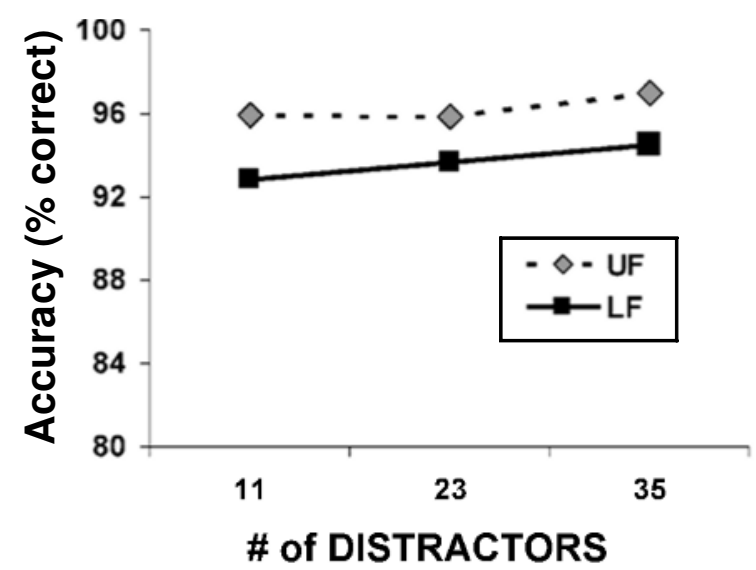

\section{ROTATION}

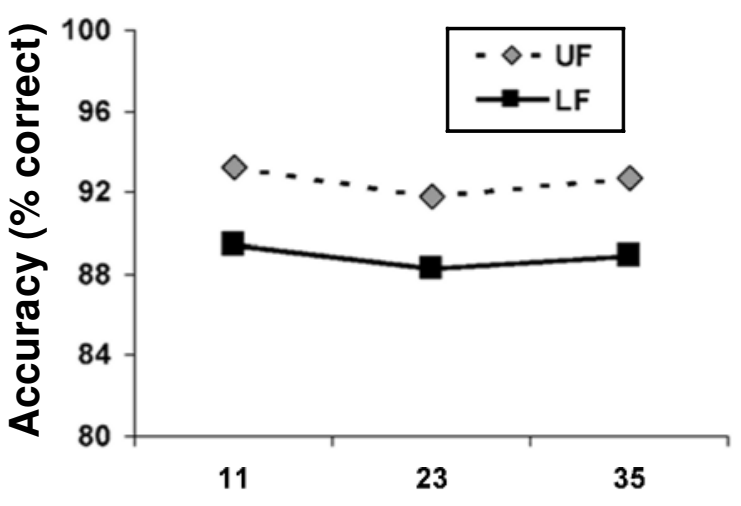

\# of DISTRACTORS

Figure 3. Percentage of correct location responses for upper field (UF, dashed lines) and lower field (LF, solid lines) targets in the tilt (left panel) and rotation (right panel) conditions of Experiment 1. The UF and LF RTs are shown for all three distractor density levels $(11,23$, and 35$)$. 


\section{CUBE TILT AND HEMIFIELD}

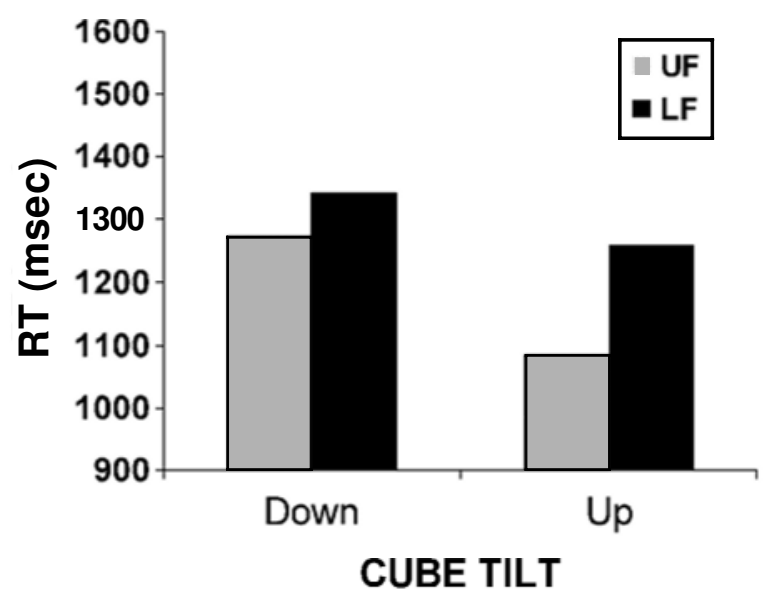

Figure 4. Mean reaction time (RT) latencies to upward-tilted targets and downward-tilted targets in the upper field (UF, gray bars) and lower field (LF, black bars) in Experiment 1.

icant tilt $\times$ hemifield interaction $[F(1,11)=22.25, p<$ $.001]$. As is shown in Figure 4, the main effect of tilt reflected the faster overall search times to upward-tilted targets $(1,182$ vs. $1,312 \mathrm{msec})$. The significant tilt $\times$ hemifield interaction reflected the fact that the RT advantage in the UF was significantly greater for upwardtilted targets than for downward-tilted targets $(186 \mathrm{msec}$, as compared with $74 \mathrm{msec}$, respectively).

\section{Discussion}

The major finding of Experiment 1 was that subjects are faster and more accurate in searching for cubes located in the upper half of a search display when the cubes are defined by their tilt and rotation in space. A UF advantage was found regardless of the type of feature or the particular tilt of the cube (upward or downward).

The superior search performance in the UF for tilt and rotation was somewhat unexpected, given the general tendency for such features to be more associated with LF-biased peripersonal (near-body) visual processing (see Previc, 1998) and von Grünau and Dubé's (1994) finding of a significant LF advantage in one of their tiltsearch experiments (Experiment 4.2). One possible reason for the UF advantage in this study is that the subjects cued more onto the local form elements - that is, the specific pattern of light and dark regions-contained in the target, which clearly differed not only for the oppositely tilted targets and distractors (see Figure 1), but also for the oppositely rotating ones at a given point in time (particularly since the distractor cubes all started from the same position). By searching for local shape information, the UF-biased focal extrapersonal attentional system that is used in object recognition (see Previc, 1998) could have been thereby activated. However, local form information was always changing as the cubes rotated in space and so could not have easily been used by the subjects as they continuously searched the display. Moreover, the subjects in von Grünau and Dubé's study may also have accessed local size or luminance information, especially when shaded 3-D polygons were presented (as in their Experiment 4). ${ }^{2}$ Finally, the large asymmetry in finding upwardtilted versus downward-tilted cubes implies that the subjects may have at least somewhat attended to the overall 3-D shape of the target, rather than just to its local form elements, given the alleged role of three-dimensionality in determining such differences (Sun \& Perona, 1996; von Grünau \& Dubé, 1994, Experiment 1).

A more likely explanation is that the UF bias during free-scan search is less dependent on the type of stimulus being searched for than on the visual search mechanism used. In this study, the subjects searched for cues that are often encountered in peripersonal space, but the search tasks they engaged in were highly similar to those that had previously yielded UF advantages. It is possible, then, that the UF bias was guaranteed by the activation of the saccadic-linked focal extrapersonal attentional system required to perform the search (Previc, 1998). This explanation can explain why mostly nonsignificant verticalhemifield differences were found by von Grünau and Dubé (1994). In that study, subjects were not allowed to make any eye movements while searching for the target, whereas the present study used a free-scan search task with eye movements permitted. Although oculomotor asymmetries cannot account for the UF search bias in its entirety (see Previc, 1996), the UF search advantage is often greatly reduced or reversed when subjects are not allowed to make any eye movements (He, Cavanagh, \& Intriligator, 1996; Previc, unpublished data ${ }^{3}$; von Grünau \& Dubé, 1994). By restraining their eye movements, von Grünau and Dubé's subjects may not have sufficiently activated the focal extrapersonal attentional system and its UF bias (see the General Discussion section).

Another noteworthy aspect of the results of Experiment 1 is that search times were, on average, more than twice as long as those in previous studies using a comparable subject population and highly similar conditionsfor example, feature search with similarly sized targets and search fields (Previc, 1996; Previc \& Blume, 1993). The search times in the tilt-search condition in Experiment 1 were also far longer than those of Enns and Rensink (1990) and von Grünau and Dubé (1994), which used similar tilted cubes as targets. One major reason for the increased overall search times in Experiment 1 relative to previous studies may have been that the cube targets were always moving leftward or rightward, even when tilt was the feature searched for; hence, the ever-changing view of the cube may have made the cube's tilt more difficult to process. An alternative explanation again relates to the nature of the task used: Enns and Rensink (1990) and von Grünau and Dubé used a present-absent (PA) task in which the target remained constant throughout a block and subjects were instructed to maintain fixation, whereas a 4ASFC, free-scan task in which the target stimulus changed from trial to trial was used in Experiment 1. Many studies have actually reported decreased search times 
when eye movements are not permitted (Carrasco, Evert, Chang, \& Katz, 1995; Previc, 1996; Zelinsky \& Sheinberg, 1997), and they are also shorter when the target does not vary from trial to trial (Bravo \& Nakayama, 1992; Findlay, 1997).

Although search times were much longer in the tilt condition of Experiment 1 than in previous tilt manipulations, they were less affected by distractor density. In von Grünau and Dubé's (1994) study, for example, the average RT to oppositely tilted cube targets ranged from 500 to $600 \mathrm{msec}$ when no distractors were present to slightly longer than $700 \mathrm{msec}$ when 12 distractors were present (see their Figure 2). By contrast, search times for oppositely tilted cubes in this study were over $1 \mathrm{sec}$ in all conditions and increased by only $17 \mathrm{msec}$ as the number of distractors increased from 11 to 35 . It is possible that the flat slopes evidenced in Experiment 1 may have been caused by the three-dimensionality of the cube stimuli, as hypothesized by both Enns and Rensink (1991) and von Grünau and Dubé and as suggested by the data reported by Sun and Perona (1996). The relatively slow but still flat-sloped search times as a function of distractor density clearly demonstrate that overall search time is not always a good predictor of the effects of distractor density (see also Wolfe, 1992). Indeed, the steep-sloped searches for size and shape cues in previous conjunction-search tasks from this laboratory (Previc, 1996; Previc \& Blume, 1993) were performed much faster than were the flat-sloped searches of Experiment 1.

A final issue that must be addressed is the difference in processing upward-tilted versus downward-tilted cubes. As in previous studies (Braun, 1993; Enns \& Rensink, 1990; Kleffner \& Ramachandran, 1992, Experiment 2; Sun \& Perona, 1996), it proved significantly easier to locate targets whose darkest regions were on top (i.e., the upward-tilted cubes) among distractors whose lightest region was on top (i.e., the downward-tilted cubes) than vice versa. This trend was obtained for both vertical hemifields, but the upward-tilted advantage was especially pronounced in the UF. By contrast, von Grünau and Dubé (1994) reported a search advantage for their upwardtilted cubes primarily in the lower visual field, which they attributed to the normal ecological view of objects from above and the consequently greater salience of targets that contradict that ecological prevalence (i.e., upwardtilted cubes). One possible explanation for the different hemifield asymmetries in the two studies is the competition between two different ecological cues-viewpoint (usually from above) versus lighting source (also typically from above). Enns and Rensink (1990) found that viewing direction was less salient than lighting asymmetry, which would fit with this study's finding that upwardtilted cubes (whose top portions were always darker) were responded to faster than downward-tilted cubes (whose top portions were always lighter) in both vertical hemifields. In contrast, von Grünau and Dubé used target cubes that had either no lighting gradients associated with them (i.e., the wire-frame cubes in their Experiments 1-3) or a common lighting-from-above feature (i.e., the pictorial 3-D depictions in their Experiment 4).

In summary, Experiment 1 showed that a UF advantage exists when subjects engage in free-scan search for cubes defined by their tilt or rotation in space. Experiment 1 also showed that search for these features is not greatly affected by distractor density, even though it is comparatively slow relative to search for object-related features. Finally, the results of Experiment 1 are consistent with those of previous cube-search studies in that upwardtilted cubes were processed faster than downward-tilted ones. The faster search for upward-tilted cubes reported by von Grünau and Dubé (1994) only in the LF was not obtained, however, possibly owing to the different lighting parameters of the two studies or to the different tasks used.

\section{EXPERIMENT 2 Effect of Lighting Direction and Tilt on Search for 3-D Objects}

Owing to a number of procedural variations between earlier cube-search studies and Experiment 1 (e.g., uniformity of lighting direction, local luminance variations, cube rotation, and type of task), additional experiments were designed to control for most of these factors. In Experiment 2, static 3-D solids were presented as targets and were, in one condition, subjected to a common direction of lighting, as in von Grünau and Dubé (1994). A common lighting source could conceivably create an ecologically prevalent condition that would favor the LF in at least one condition-namely, when the distractor field is tilted downward and the light emanates from above, as normally occurs when viewing the ground in daytime (see von Grïnau \& Dubé, 1994). An LF search advantage obtained under this condition would highlight the contribution of specific ecological factors to vertical-hemifield biases in 3-D target search. If a UF search advantage were to hold even in this condition, however, it would further confirm that UF biases are less dependent on the nature of the stimuli than on the nature of the search process.

When creating a common lighting direction, it is necessary to use 3-D rectangular solids (truncated cubes) in order to eliminate luminance artifacts. This is because the top or bottom of a cube tilted at $25^{\circ}$ relative to the observer subtends less total area than do the two sides of the cube; hence, a common lighting source from above that lights up the top of a downward-tilted cube and the sides of an upward-tilted cube results in a brighter overall target in the latter case. Luminance artifacts are eliminated only when the sides of a tilted cube are reduced to a combined area equal to that of its top or bottom.

\section{Method}

Subjects. A total of 12 subjects, 10 of whom were male, participated in Experiment 2. The age of the subjects ranged from 18 to 59 years, with a mean age of 36.0 years. All the subjects were either full-time or visiting civilian and military personnel at Brooks Air Force Base. None had participated in Experiment 1 or any other 


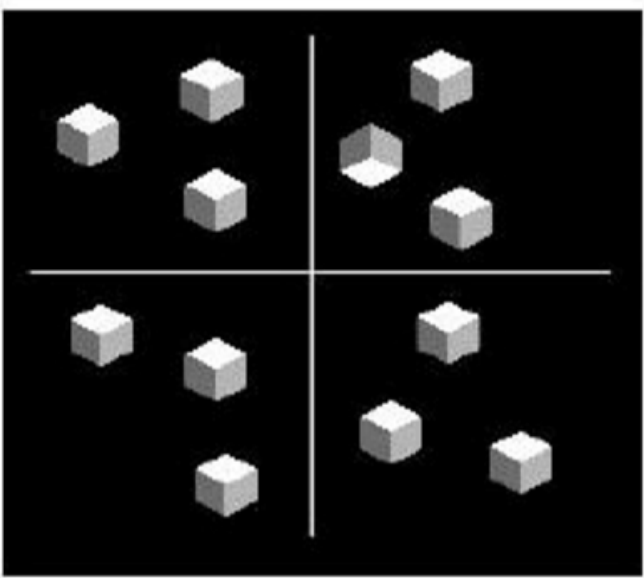

CUBE

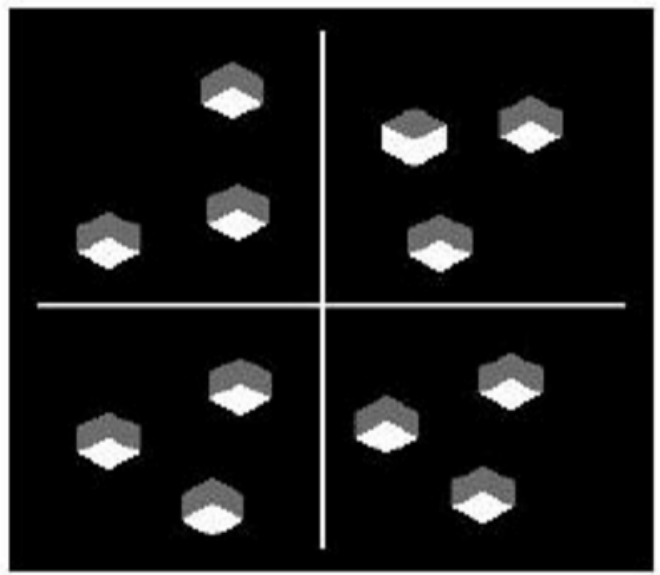

RS/UL

Figure 5. The two stimulus types used in Experiment 2: full-sized static cubes (CUBE) and rectangular solids with uniform lighting (RS/UL). The dimensions and contrasts portrayed are not identical to those of the actual stimuli.

previous visual search study and all were naive as to the hypotheses of the study. Each subject had 20/30 visual acuity or better, with or without correction, in each eye.

The voluntary, informed consent of subjects was obtained as required by Air Force Instructions 40-402 and 40-403.

Stimuli and Apparatus. There were two stimulus conditions in Experiment 2, shown in Figure 5. The first of these conditions, termed $C U B E$, presented static cubes pitched either upward or downward $25^{\circ}$ with a nonuniform lighting source (i.e., the upwardtilted cube was effectively lit from below, with different shades of gray for the two sides, whereas the downward-tilted cube was effectively lit from above in the same manner). The second of these conditions, termed $R S / U L$, presented upward- and downward-tilted rectangular solids as targets and distractors; these were lit uniformly, either from above or below on a given trial. The stimuli in the CUBE condition were highly similar to those used in Experiment 1 , but they even more closely resembled the cube stimuli used by Enns and Rensink (1990) and Sun and Perona (1996), because their motion was eliminated. The RS/UL stimuli were formed by truncating the CUBE stimuli into rectangular solids, the combined area of whose sides equaled that of the top or bottom. Although the average luminance of the RS/UL stimuli did not vary when they were tilted upward or downward relative to a common lighting source, they also did not appear as 3-D as the CUBE stimuli. ${ }^{4}$

Each side of the CUBE stimuli subtended $0.8 \mathrm{~cm}$. The rectangular solids subtended the same visual angle as the cube, except that their height was $40 \%$ of the cube's height. The average luminance of all 3-D shapes was $7.18 \mathrm{~cd} / \mathrm{m}^{2}$, whereas that of the background was $0.06 \mathrm{~cd} / \mathrm{m}^{2}$. The contrast between the lightest and the darkest regions of the CUBE and RS/UL solids was 0.71 . The luminance of one of the two sides of the CUBE stimulus was equal to the average luminance of the lighter top (or bottom) of the cube and the other darker-depicted side. The two sides of the RS/UL stimulus, on the other hand, were equiluminant and could be brighter or dimmer than the top or bottom, depending on the direction of lighting.

All the stimuli were generated on a Silicon Graphics Indigo 2 computer and displayed on a Sony $60-\mathrm{Hz}$ color monitor (Model GDM-20E21). The viewing distance of $81.3 \mathrm{~cm}$ was identical to that of Experiment 1, as was the overall field size of the display $\left(12.3^{\circ}\right)$ and the centers of the three eccentricity rings $\left(2.1^{\circ}, 3.6^{\circ}\right.$, and $\left.5.6^{\circ}\right)$. The subjects viewed the stimuli in a darkened room while sit- ting on a chair that was adjustable in height, and they rested their forehead and chin in an ophthalmologic viewing brace. The subjects responded to the target stimulus using a mouse (Mouse Systems Model 4Q) that was attached to the Indigo 2 computer.

Task and Overall Procedure. The main task performed by all the subjects was a search for upward- or downward-tilted 3-D targets among 11 oppositely tilted distractors (3 per quadrant, except in the quadrant in which the target appeared). This task required essentially the same 4ASFC RT response as that in Previc and Blume (1993), Previc (1996), and Experiment 1. An individual trial began with a 1 -sec presentation of the centrally located circle and was then followed by the appearance of the circle and an enclosed small fixation cross for $500 \mathrm{msec}$. The fixation cross and circle were then replaced for $200 \mathrm{msec}$ by one of the two tilted 3-D cue targets. The subject had up to $3 \mathrm{sec}$ to find the target in the search field, using a free-scan strategy, and as long as $2 \mathrm{sec}$ to move the cursor to the correct quadrant in the postsearch display. Both the detection and the location responses were made on the left key of the mouse, using the index finger of the right hand.

As in Experiment 1, a visual search trial block consisted of a total of 144 trials. Each of the two target cubes appeared six times in each eccentricity ring in each quadrant throughout a trial block, using the same pseudorandom sampling-without-replacement as in Experiment 1 . The subject could pause the trial sequence at any time by pressing the rightmost key on the mouse.

The experiment was run over four 30-45 min sessions, each held on a different day. In the two training sessions, the CUBE and RS/UL stimuli were presented for three trial blocks each (for a total of six trial blocks overall). In each of the two test sessions, each stimulus condition was presented once, for a total of two replications overall. The order of presentation of the two stimuli was alternated across subjects.

\section{Results}

The results of Experiment 2 were subjected to repeated measures ANOVAs for both speed (RT) and accuracy (percentage correct). Three within-subjects factors were analyzed: stimulus condition (CUBE vs. RS/UL), vertical hemifield (UF vs. LF), and target tilt (upward vs. downward). Because the direction of lighting in the 


\section{SPEED}
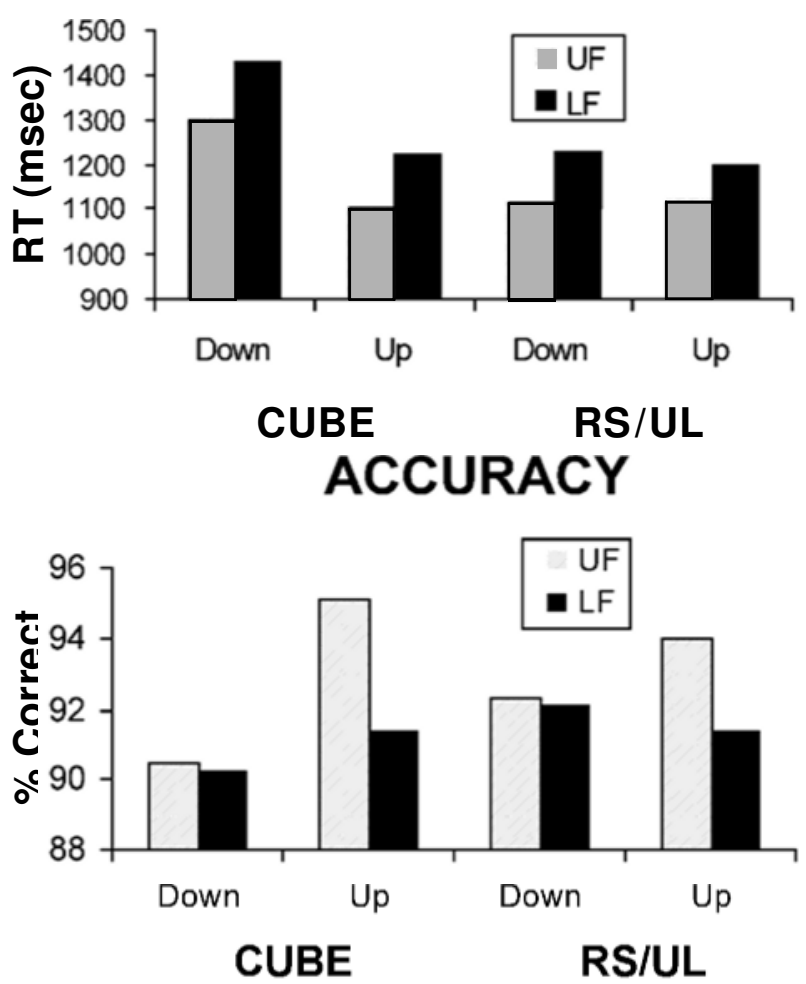

Figure 6. Top: Mean reaction time (RT) latencies in Experiment 2 as a function of stimulus type (full-sized static cube [CUBE] vs. rectangular solids with uniform lighting [RS/UL]), target tilt (downward or upward), and vertical hemifield (upper field [UF], represented by gray bars; lower field [LF], represented by black bars). Bottom: Percentage of correct location responses in Experiment 2 as a function of stimulus type (CUBE vs. RS/UL), target tilt (downward or upward), and vertical hemifield (UF, represented by gray bars; LF, represented by black bars).

CUBE condition did not vary but, instead, always showed the upward-tilted cube lit from above and the downwardtilted cube lit from below, subsequent RT and accuracy ANOVAs that included the lighting factor were performed only on the RS/UL data.

Three-factor ANOVAs. The three-factor speed ANOVA, based on the RT means shown in the top panel of Figure 6, revealed only a significant main effect of vertical hemifield $[F(1,11)=9.48, p<.01]$ and a significant stimulus condition $\times$ tilt interaction $[F(1,11)=15.75$, $p<01]$. The vertical-hemifield main effect reflected the faster RTs in the UF $(1,160 \mathrm{msec})$, as compared with the $\mathrm{LF}(1,273 \mathrm{msec})$. The condition $\times$ tilt interaction reflected the much faster RTs $(1,165$ vs. $1,369 \mathrm{msec})$ to upwardtilted than to downward-tilted targets in the CUBE condition, as opposed to the negligible advantage for the upward-tilted targets in the RS/UL condition (1,160 vs. $1,172 \mathrm{msec}$ ).
The three-factor accuracy ANOVA, based on the means of the percentages of correct responses shown in the bottom panel of Figure 6, revealed no significant effects at $p<.05$. However, the vertical hemifield and condition $\times$ tilt trends were in the same direction as in the RT data. For example, accuracy was greater in the UF $(93.0 \%$ vs. $91.3 \%$ ), and there was a greater upward-tilt accuracy advantage in the CUBE condition $(93.2 \%$ vs. $90.4 \%)$ than in the RS/UL one ( $92.6 \%$ vs. $92.3 \%$ ). Thus, there was no evidence of a speed-accuracy tradeoff for either of these effects.

Lighting ANOVAs. The effect of lighting direction on RTs in the RS/UL condition is shown in the top panel of Figure 7. There was a significant main effect of vertical hemifield $[F(1,11)=4.79, p=.05]$ in the RT ANOVA, reflecting a 100-msec RT advantage for the UF, as well as a significant interaction between target tilt and lighting direction $[F(1,11)=27.312, p<.001]$. This interaction reflected the faster RTs to upward-tilted targets lit from above, as compared with below (1,069 vs. 1,274 msec), in contrast to the faster RTs to downward-tilted targets when lit from below, as compared with above $(1,059$ vs. $1,261 \mathrm{msec})$. There was a similar tilt $\times$ lighting interaction in the accuracy ANOVA $[F(1,11)=7.92, p<.05]$, with accuracy better for upward-tilted targets when lit from above, as compared with below (94.2\% vs. 90.3\%) and for downward-tilted targets when lit from below, as compared with above $(94.1 \%$ vs. $91.2 \%$; bottom panel of Figure 7). There was also a significant vertical hemifield $\times$ lighting interaction $[F(1,11)=17.84, p=.001]$, which reflected a greater accuracy for targets lit from above, as compared with below, in the UF $(94.4 \%$ vs. $91.9 \%$ ), as opposed to a greater accuracy for targets lit from below in the LF (92.5\% vs. $91.0 \%)$.

\section{Discussion}

The results of Experiment 2 are noteworthy in four principal respects. First, the data from the CUBE condition generally replicate the tilt results of Experiment 1 in terms of overall mean RT and the magnitude of the UF RT advantage. Second, the UF advantage in free-scan search has been shown to extend to yet another type of stimulus-tilted rectangular solids. Third, the UF advantage for 3-D targets was not shown to be influenced by the direction of lighting, although lighting and target tilt did themselves interact. Finally, the overall search bias in favor of upward-tilted cubes was replicated only in the CUBE condition, thereby suggesting that this bias may be partly related to some 3-D property of the target and/or distractor field.

The mean RT in the CUBE condition was $1,267 \mathrm{msec}$ in Experiment 2, as compared with 1,088 $\mathrm{msec}$ in the tilt condition of Experiment 1 that had the same number of distractors (11) and identical (albeit rotating) targets. As was noted earlier, both of these RT values are over $500 \mathrm{msec}$ longer than the mean search times for objectrelated features, such as size and shape, under virtually 


\section{SPEED}

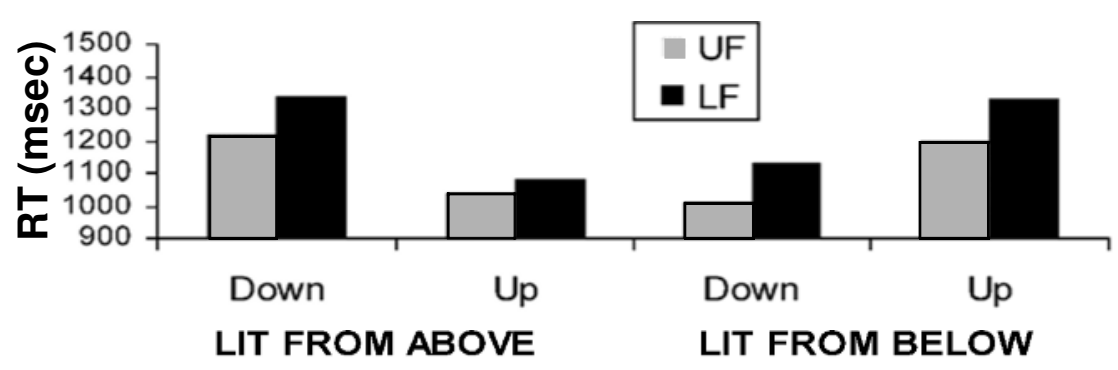

\section{ACCURACY}

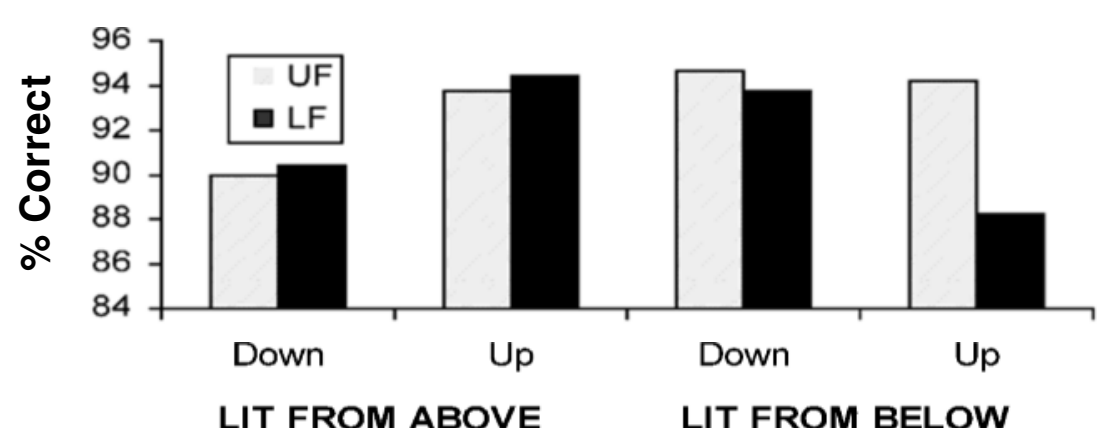

Figure 7. Top: Mean reaction time (RT) latencies in the RS/UL condition of Experiment 2 as a function of target tilt (downward or upward), vertical hemifield (upper field [UF], in gray bars; lower field [LF], in black bars), and direction of lighting (lit from above vs. lit from below). Bottom: Percentage of correct location responses in the RS/UL condition of Experiment 2 as a function of target tilt (downward or upward), vertical hemifield (UF, in gray bars; LF, in black bars), and direction of lighting (lit from above vs. lit from below).

identical visual and task conditions. Unlike in Experiment 1, the long RTs in Experiment 2 cannot be ascribed to the continuous rotation of the stimulus. Rather, these results indicate that 3-D target information may not be as salient for the visual search system as are highly distinctive cues, such as color and 2-D orientation (see Brown et al., 1992). Although Enns and Rensink (1990) and von Grünau and Dubé (1994) reported much shorter RTs in their 3-D search experiments, this may be due to such factors as type of task, target predictability, and restriction of eye movements (see the previous discussion).

It is also noteworthy that both sets of target stimuli in Experiment 2 were found much more quickly when they were located in the upper half of the search field. The magnitude of the UF RT advantage ( 100 msec) was similar to that found in Experiment 1 and was even greater than that obtained in size-shape search studies under similar conditions (Previc, 1996; Previc \& Blume, 1993). The UF search advantage was also present for both directions of lighting in the RS/UL condition. Thus, the UF advantage in free search appears relatively insensitive to such stimulus factors as 3-D appearance, rotation, uniformity of lighting, and direction of lighting. As with the decreased overall search times, the absence of an UF advantage in von Grünau and Dubé's (1994) study appears to be more related to the type of search task and restriction of eye movements than to the nature of the target stimulus per se.

Lighting direction did exert a powerful influence on the ability to find upward-tilted versus downward-tilted RS/UL targets. Upward-tilted targets lit from above were much more quickly located than were downward-tilted targets lit from above, and vice versa. Although this finding lends nominal support to the ecological argument, in that objects lying along the downward-sloping ground plane are more likely to be lit from above (thereby increasing the salience of similarly lit upward-tilted targets), there may be an alternative explanation for this finding. Many subjects reported that the stimuli in the RS/UL condition appeared more like 2-D chevrons or diamonds than 3-D rectangular solids (see note 4). When lit from above, the upward-tilted solid appeared chevron-like and 
the downward-tilted solid appeared diamond-like, whereas the reverse was the case for solids lit from below. Hence, the tilt $\times$ lighting interaction could have been obtained merely if chevrons are inherently easier to perceive than diamonds. In any case, the lack of a significant overall advantage for upward-tilted solids in the RS/UL condition demonstrates that the ecological salience of dark-topped, upward-tilted shapes against a downward-tilted distractor field is not automatically registered for shapes that do not have a good 3-D appearance.

The advantage of the upward-tilted cubes in the CUBE condition did replicate previous cube-search findings, including those from the tilt condition of Experiment 1 and from Sun and Perona (1996) and von Grünau and Dubé (1994). It is unlikely that the upward-tilted bias in this case was merely a consequence of the tilt $\times$ lighting interaction demonstrated in the RS/UL condition, because the upward-tilted cube was lit from below in the CUBE condition, whereas upward-tilted targets in the RS/UL condition were more quickly found when they were lit from above. Rather, the specific advantage of the upwardtilted cubes would seem to be more related to their 3-D appearance (see also Sun \& Perona, 1996, and the General Discussion section).

\section{EXPERIMENT 3 Vertical Field Asymmetries in Spatial Forced-Choice Versus Present-Absent Search Tasks}

As was noted in the preceding discussion, one explanation for the longer overall search times and robust UF advantages obtained in Experiments 1 and 2, relative to those of Enns and Rensink (1990) and von Grünau and Dubé (1994), is the type of task used. Whereas Enns and Rensink (1990) and von Grünau and Dubé used a PA task, Previc and Blume (1993) and Previc (1996) used the same 4ASFC task as that used in Experiments 1 and 2 of this study. Although Zelinsky (1996) also obtained upward oculomotor and search biases by using a PA task, his targets were colored bars and not 3-D shapes. Therefore, the purpose of Experiment 3 was to test whether verticalhemifield biases in free-scan search for 3-D shapes are affected by the use of a SFC versus PA search task.

\section{Method}

Subjects. The subject population consisted of 6 of the 12 subjects who participated in Experiment 2.

Stimuli and Apparatus. The stimuli used in this experiment were identical to those in the CUBE condition of Experiment 2, and they were presented using the same apparatus. The timing of the fixation, cue, and search field presentations, along with the locations of the target stimuli, were also identical to those of Experiment 2 .

Task and Overall Procedure. The task used in this experiment was a PA search task in which the target stimulus appeared on only half the trials. A trial block consisted of 288 trials, which meant that the same number of target-present trials (144) occurred in both the 4ASFC task of Experiments 1 and 2 and the PA task of Experiment 3 . The subjects were required to press the left mouse button on trials in which the target appeared and the middle button on trials in which it did not, both of which responses immediately terminated the search field. (The rightmost mouse key was used for pausing the trial block.) Although there were twice as many trials as in Experiments 1 and 2, the fact that no location response was required at the termination of the search field kept the overall trial-block length to about $10 \mathrm{~min}$.

The subjects received two PA practice blocks on one day and a third practice block and a single block of PA test trials on a different day.

\section{Results}

The RT data from the target-present trials of the PA task are presented in Figure 8, alongside the data from the comparable CUBE condition of Experiment 2 for the 6 subjects who performed both tasks. The mean search RT was over $150 \mathrm{msec}$ faster in the PA task $(1,063 \mathrm{msec})$ than in the 4ASFC task $(1,239 \mathrm{msec})$, and overall accuracy similarly improved in the PA task (94.2\% vs. 89.9\%). However, similar UF advantages were obtained for both tasks in terms of speed $(\Delta \mathrm{RT}=-117 \mathrm{msec}$ for PA; $\Delta \mathrm{RT}=$ $-82 \mathrm{msec}$ for 4ASFC) and accuracy $(\Delta+2.4 \%$ for PA; $\Delta$ $+1.6 \%$ for 4 ASFC). Repeated measures ANOVAs with two within-subjects factors (vertical hemifield and task) were performed on the RT and accuracy data. The RT ANOVA revealed significant effects of vertical hemifield $[F(1,5)=6.90, p<.05]$ and $\operatorname{task}[F(1,5)=7.57, p<.05]$, but no significant interaction between them. No significant main effects or interactions were found in the accuracy ANOVA.

\section{Discussion}

The use of PA search tasks in previous 3-D search studies versus the use of SFC tasks in Experiments 1 and 2 cannot account for the discrepant findings between the two sets of studies in terms of overall search speed or vertical-hemifield asymmetries. Although search times were considerably faster in the PA task of Experiment 3 than in the SFC task of Experiment 2, they were still several hundred milliseconds longer than in the PA tasks used by Enns and Rensink (1990) and von Grünau and Dubé (1994). More important, the UF advantage in the PA task was similar to that in the SFC task of Experiment 2, whereas von Grünau and Dubé found a significant LF advantage for upward-tilted targets when a uniform lighting source was used in conjunction with their PA task (in their Experiment 4.2). This difference points to two other procedural differences between the present study and those of Enns and Rensink (1990) and von Grünau and Dubé-namely, the trial-to-trial variation in the target stimulus and the opportunity to make eye movements. The pseudorandom designation of targets on a particular trial would seem to be unimportant in fostering the UF advantage, in that Zelinsky (1996) also obtained free-scan UF oculomotor and performance biases when the search target was fixed for an entire trial block, although the target unpredictability may have served to lengthen overall search times (Bravo \& Nakayama, 1992; Findlay, 1997). Rather, it would seem that the use of a 


\section{SPATIAL FORCED-CHOICE VS. PRESENT-ABSENT TASK}
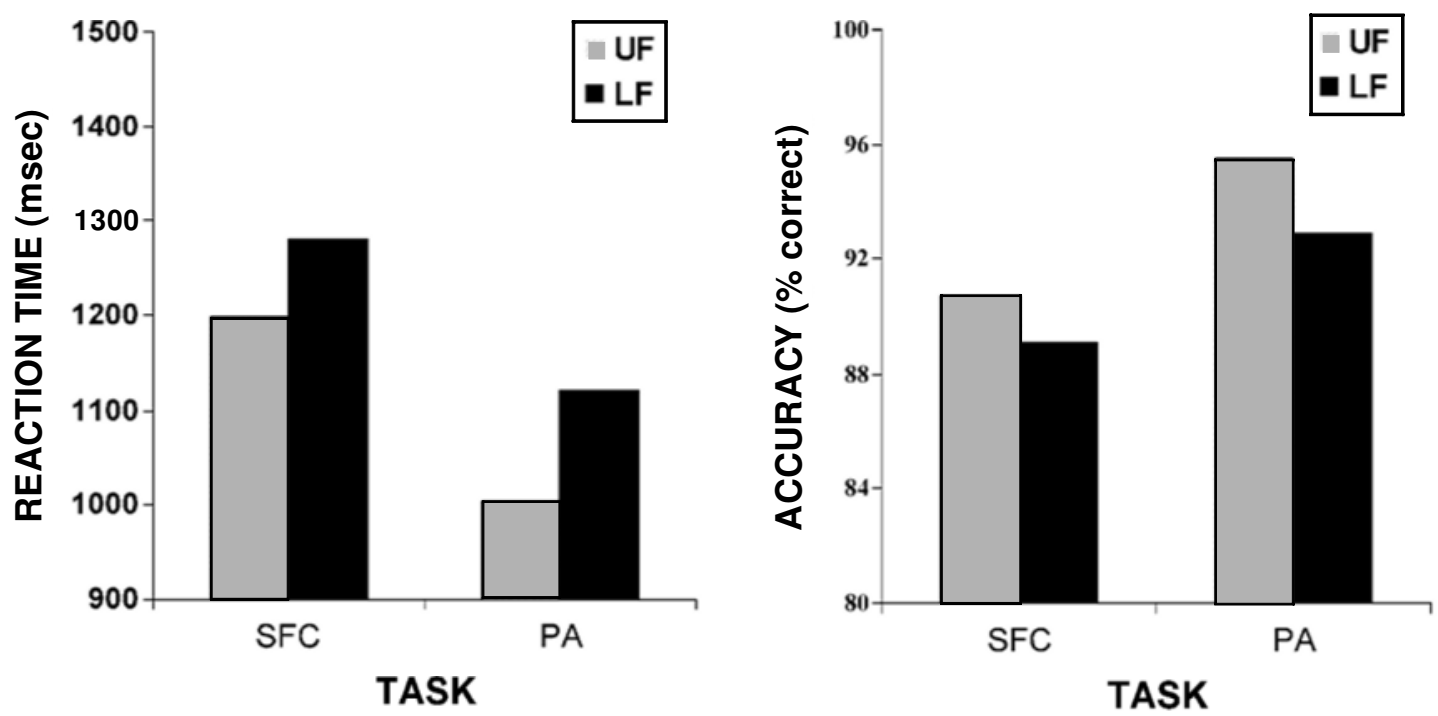

Figure 8. A comparison of mean reaction time (RT) latencies (left panel) and percentage of correct location responses (right panel) in the upper field (UF, gray bars) and lower field (LF, black bars) for the spatial forced-choice (SFC) and present-absent (PA) tasks of Experiment 3.

free-scan strategy by our subjects represents the major source of the UF advantage in searching for both 2-D and 3-D targets.

\section{GENERAL DISCUSSION}

The results of these and previous experiments support the following conclusions concerning visual search for the tilt and rotation of cubes and other 3-D shapes: (1) Freescan search for 3-D features exhibits a UF advantage similar to that of search for 2-D object-related cues; (2) search for 3-D features is generally slower than is search for 2$D$ features, such as size, shape, and color; and (3) the bias in finding upward- versus downward-tilted shapes may be partly dependent on the 3-D appearance of the shapes.

The UF advantage for 3-D shapes was found in all the conditions of Experiments 1, 2, and 3, with its magnitude ranging from 82 to $156 \mathrm{msec}$. These advantages were, if anything, slightly greater than the UF advantage for 2-D features under similar conditions (Previc, 1996). Thus, it may be concluded that the UF bias in free search is not critically influenced by the type of target being searched for. Nor does the UF bias appear to be affected by the nature of the task, as long as eye movements are permitted to be made in finding the target. Although UF advantages can be obtained even on free-scan trials when eye movements are not made (Previc, 1996, Experiment 2) or when the search field disappears before an eye movement is made (Chaiken, Corbin, \& Volkmann, 1962), they do not occur whenever subjects are not permitted to make eye movements at all (e.g., He et al., 1996; Previc, unpublished data; von Grünau \& Dubé, 1994). These findings can be explained by the fact that the focal extrapersonal attentional system - which is used in visual search and is reputedly biased toward distal space and the UF (Previc, 1998) - is activated in preparation for an eye movement even if the latter is not subsequently made or is made after the search field is turned off (see Deubel \& Schneider, 1996; Previc, 1996). Indeed, the neuroanatomical projection stream of the focal extrapersonal visual system is closely linked to the saccadic scanning centers found in the frontal and parietal eye-fields (Previc, 1998). Shifting of attention increases with the probability of making an eye movement, which explains why the UF RT advantage in these and previous experiments generally expands with increasing search difficulty (Previc, 1996). For example, feature search for 2-D size or orientation yields mean RTs of $\sim 500 \mathrm{msec}$ and UF advantages of $\sim 50 \mathrm{msec}$ (Previc, 1996), whereas UF RT biases of over $100 \mathrm{msec}$ were associated with overall mean RTs of $>1,000 \mathrm{msec}$ in the present study.

The type of target to be searched for appears to more greatly affect overall search times, in that search for such features as tilt and rotation in space is much slower under the same task conditions and target and field sizes than is search for 2-D object-related cues, such as size, shape, and color. As was previously noted, searching for 3-D tilt or rotation in Experiments 1 and 2 yielded approxi- 
mately doubled search times, relative to those for size and 2-D orientation in previous studies (Previc, 1996; Previc $\&$ Blume, 1993). Thus, in contrast to the conclusions of Enns and Rensink (1990), von Grünau and Dubé (1994), and Sun and Perona (1996), our data suggest that the visual search system is not ideally designed to process such 3 -D features. ${ }^{5}$ Two caveats must be added to the above conclusion, however. First, search for 3-D shapes defined by their tilt in space has been shown to be faster than search for 2-D shapes when the latter are composed of an arbitrary arrangement of the same shape and/or luminance elements (Enns \& Rensink, 1990; Sun \& Perona, 1996; von Grünau \& Dubé, 1994). Hence, the 2-D advantage may only exist for shapes that are normally processed by the visual system. Second, all previous studies (including Experiment 1 of the present one) have shown that search for cubes defined by their tilt in space is relatively independent of the number of distractors. That a cue should exhibit such independence, however, does not necessarily indicate that it is ideal for the visual search system (see the previous discussion).

The final issue to be addressed is why a specific search advantage exists for upward-tilted cube targets, as in the tilt condition of Experiment 1, the CUBE condition of Experiment 2, and previous cube-search studies (Sun \& Perona, 1996; von Grünau \& Dubé, 1994). Previous explanations have focused on the contributions of lighting (Enns \& Rensink, 1990), observer view-angle (von Grïnau \& Dubé, 1994), and 3-D appearance (Sun \& Perona, 1996), but none of these explanations alone is entirely satisfactory. The lighting explanation argues that because the normal lighting direction in our visual environment is from above, stimuli that are darker on top deviate from the typically encountered luminance gradient and are, therefore, more perceptually salient (Braun, 1993; Enns \& Rensink, 1990; Kleffner \& Ramachandran, 1992). This explanation can account for the results of Experiment 1, the CUBE data of Experiment 2, and the results of Sun and Perona (1996), because the upward-tilted cubes were darker on top in all of these cases. However, the lighting explanation is insufficient because an upward-tilted bias was found by von Grünau and Dubé (1994) for their wireframe cubes with no shading and because an upwardtilted bias was found in the RS/UL condition of Experiment 2 only when the target was lit from above (and, therefore, lighter on top). The view-angle hypothesis is based on the fact that upward-tilted objects stand out against a ground-plane that normally slopes downward toward us in the LF (von Grünau \& Dubé, 1994). However, the LF advantage of von Grünau and Dubé's upwardtilted stimuli was significant in only one of their four experiments, and this explanation cannot account for the advantage of upward-tilted cubes in both visual fields in Experiments 1 and 2 of the present study. Finally, the 3-D explanation argues that the upward-tilt bias occurs because upward-tilted cubes appear more 3-D-like (Sun \& Perona, 1996). This hypothesis can account for the upward-tilt bias for the cube stimuli of Sun and Perona (1996, Figure 7), the wire-frame and rectangular solid stimuli of von Grünau and Dubé, and the cube stimuli of Experiments 1 and 2. However, an upward-tilted bias was also observed for Sun and Perona's "pie-shaped" targets, which were not rated as highly $3-D$ by our subjects (see note 1). It is likely, however, that three-dimensionality does contribute to the advantage of upward-tilted shapes, especially in interaction with lighting direction. ${ }^{6}$

In summary, the results of this study are best explained by resorting to the ecological specialization of the visual search system for distant (and consequently, UF) features-particularly those that would be valuable in helping to find and recognize relevant objects in the environment (see Previc, 1998). Although other features, such as the 3-D orientation and rotation of the target stimulus and direction of lighting, appear to be registered by the visual search system, these other properties play only a minor role, if any, in the genesis of the UF bias in freescan visual search.

\section{REFERENCES}

Braun, J. (1993). Shape-from-shading is independent of visual attention and may be a "texton." Spatial Vision, 7, 311-322.

Bravo, M. J., \& NaKayama, K. (1992). The role of attention in different visual-search tasks. Perception \& Psychophysics, 51, 465472.

Brown, J. M., Weisstein, N., \& May, J. G. (1992). Visual search for simple volumetric shapes. Perception \& Psychophysics, 51, 40-48.

Carrasco, M., Evert, D. L., Chang, I., \& Katz, S. M. (1995). The eccentricity effect: Target eccentricity affects performance on conjunction searches. Perception \& Psychophysics, 57, 1241-1261.

Chaiken, J. D., Corbin, H. H., \& Volkmann, J. (1962). Mapping a field of short-time visual search. Science, 138, 1327-1328.

Deubel, H., \& Schneider, W. X. (1996). Saccade target selection and object recognition: Evidence for a common attentional mechanism. Vision Research, 36, 1827-1837.

Enns, J. T., \& Rensink, R. A. (1990). Influence of scene-based properties on visual search. Science, 247, 721-723.

Enns, J. T., \& Rensink, R. A. (1991). Preattentive recovery of threedimensional orientation from line drawings. Psychological Review, 98, 335-351.

FINDLAY, J. M. (1997). Saccade target selection during visual search. Vision Research, 37, 617-631.

He, S., Cavanagh, P., \& Intriligator, J. (1996). Attentional resolution and the locus of visual awareness. Nature, 383, 334-337.

Humphreys, G. W., Keulers, N., \& Donnelly, N. (1994). Parallel visual coding in three dimensions. Perception, 23, 453-470.

Kleffner, D. A., \& Ramachandran, V. S. (1992). On the perception of shape from shading. Perception \& Psychophysics, 52, 18-36.

Previc, F. H. (1996). Attentional and oculomotor influences on visual field anisotropies in visual search performance. Visual Cognition, $\mathbf{3}$, 277-301.

Previc, F. H. (1998). The neuropsychology of 3-D space. Psychological Bulletin, 124, 123-164.

Previc, F. H., \& Blume, J. L. (1993). Visual search asymmetries in three-dimensional space. Vision Research, 33, 2697-2704.

Sun, J. Y., \& Perona, P. (1996). Preattentive perception of elementary three-dimensional shapes. Vision Research, 36, 2515-2529.

von GrünaU, M., \& DubÉ, S. (1994). Visual search asymmetry for viewing direction. Perception \& Psychophysics, 56, 211-220.

Wolfe, J. M. (1992). "Effortless" texture segmentation and "parallel" visual search are not the same thing. Vision Research, 32, 757-763.

ZELINSKY, G. J. (1996). Using eye saccades to assess the selectivity of search movements. Vision Research, 36, 2177-2187.

Zelinsky, G. J., \& Sheinberg, D. L. (1997). Eye movements during parallel-serial visual search. Journal of Experimental Psychology: Human Perception \& Performance, 23, 244-262. 


\section{NOTES}

1. Indeed, as an addendum to Experiment 2 of this study, the 12 subjects who participated in it were asked to rank eight representative stimuli from Sun and Perona (1996) - their Figures 1A, 1C, 4, 5, 6, 7, 8, and 9-for "3-D quality." The most rapidly processed of these stimuli-the "pie" shapes in their Figure 6-were ranked second-poorest by our subjects in terms of "3-D quality" (6.25 on an 8-point scale), whereas the "pyramid" stimulus in their Figure 5, which was rated second-highest in 3-D appearance, with a 2.0 rating, required considerably longer processing times to be found.

2. For example, we have calculated that the percentage of shaded area in the target versus distractor shapes differed by a factor of 2.4 in von Grünau and Dubé's (1994) study (see their Figure 4C).

3. For example, the 52-msec UF advantage in the high-density featuresearch condition of Experiment 2 of Previc (1996) was reduced to $4 \mathrm{msec}$ when subjects were prevented from making eye movements (Previc, unpublished data).

4. Using a 5-point scale, with 5 being the most 3-D like, the subjects rated the CUBE stimuli as 4.7 and the RS/UL stimuli as 2.8 .

5 . The ability to find a target consisting of a conjunction of tilt and rotation is still more difficult to perform. Even after extensive training, most subjects cannot perform a conjunction search for these features at better than $50 \%$ accuracy when the number of distractors (11) is the same as that in Experiment 2 (Previc, unpublished data). This is very different from conjunction search for size and shape cues under similar conditions, which is performed at better than 90\% accuracy (Previc, 1996; Previc \& Blume, 1993).

6. For example, the target cubes shown in Figures 1 and 5 appear to most subjects to have darker sides (and consequently, more overall contrast) when tilted upward than when tilted downward, relative to the distractor cubes. (This illusion, which is less obvious for the rectangular solids are also shown in Figure 5, can easily be demonstrated by inverting the figures.) The 3-D-lighting illusion may be caused by observers' discounting the luminance attributable to an assumed overhead light source, thereby reducing the perceived luminance of the upward-facing portions of the cube. It is unclear whether the increased perceived contrast between the light and the dark regions of the upward-tilted cube contributes very much to the faster search time for it. This illusion does, however, suggest that a combination of the ecological lighting and 3-D shape explanations may best explain the search bias in favor of upwardtilted cubes, since no overall upward-tilted bias was found in the case of the rectangular solids that appeared more 2-D.

(Manuscript received December 28, 1999; revision accepted for publication October 5, 2000.) 